\title{
A turbulence closure based on the maximum entropy method
}

\author{
R. W. Derksen \\ Department of Mechanical and Manufacturing Engineering, \\ University of Manitoba, Winnipeg, Canada
}

\begin{abstract}
The fundamental problem of turbulence is that of closing the infinite sequence of equations that result from the application of Reynolds averaging to the governing relations for momentum, heat and mass transfer. These equations model the moments of the turbulent probability density, PDF, such as the first, second, third, and higher order moments, each equation depending on higher order moments. The ability to relate the set of moments of order $n$ to moments of $n+1$ would permit closure to a finite system of equations as we could truncate the sequence of equations. The concept of the Shannon entropy allows us to model the degree of uncertainty of a PDF. The Shannon entropy is related to the concept of thermodynamic entropy. The maximum entropy method determines the PDF that maximizes the entropy subject to a number of constraints. The most usual method is to use a finite number of lower order moments. A maximum entropy PDF is often used to approximate the shape of a PDF as the solution has desirable features such as being positive definite. The maximum entropy method is of great value as an approximation method in general. An examination of the behavior of the moments generate from a maximum entropy for a single degree of freedom fit to real, turbulent PDFs for velocity, skin-friction, and temperature fluctuations have been carried out to examine the methods ability predictive capability. In this examination experimentally determined data sets that contained data for all moments up to the sixth order were compiled from the literature. The maximum entropy method was applied using the first four moments. The fifth and sixth moments computed from the maximum entropy approximations were compared and found to compare very favorably with those measured. The presentation will start with a review of the maximum entropy method for a finite number of moments and a discussion of the computational
\end{abstract}


issues that arise. This will be followed by a review of the experimental data used, and a comparison of the maximum entropy moments with the experimental data. The presentation will conclude with a presentation of the solution to the maximum entropy method for up to four degrees of freedom.

Keywords: turbulence closure, maximum entropy, turbulence modelling, turbulent PDF.

\section{Introduction}

The fundamental problem of turbulence is that of closing the infinite sequence of equations that result from the application of Reynolds averaging to the governing relations for momentum, heat and mass transfer. These equations model the moments of the turbulent probability density, PDF, such as the first, second, third, and higher order moments, each equation depending on higher order moments. The ability to relate the set of moments of order $n$ to moments of $n+1$ would permit closure to a finite system of equations as we could truncate the sequence of equations.

The maximum entropy method is of great value as an approximation method in general. An examination of the behaviour of the moments generated from a maximum entropy fit to real, turbulent PDFs for velocity, skin-friction, and temperature fluctuations have been carried out to examine the methods ability predictive capability. In this examination experimentally determined data sets that contained data for all moments up to the sixth order were compiled from the literature. The maximum entropy method was applied using the first four moments. The fifth and sixth moments computed from the maximum entropy approximations were compared and found to compare very favourably with those measured.

The presentation will start with a review of the single degree of freedom maximum entropy method for a finite number of moments and a discussion of the computational issues that arise. This will be followed by a review of the experimental data used, and a comparison of the maximum entropy moments with the experimental data. We will conclude with the development of a four degree of freedom maximum entropy solution.

\section{The Shannon entropy of a probability density function}

The concept of the Shannon entropy allows us to model the degree of uncertainty of a PDF for a random variable $U$, expressed as $p=p(U)$, through an integral given by

$$
s=-\int_{-\infty}^{\infty} p(U) \ln p(U) d U .
$$

The concept of the Shannon entropy also applies where the PDF has finite support allowing us to consider integrals with finite limits. The Shannon entropy 
is related to the concept of thermodynamic entropy as developed by Boltzmann, see Atkins [1].

The maximum entropy method determines the PDF that maximizes the entropy subject to a number of constraints. The most usual method is to use a finite number of lower order moments. A maximum entropy PDF is often used to approximate the shape of a PDF as the solution has desirable features such as being positive definite.

\section{The maximum entropy method for one degree of freedom}

The maximum entropy approximation to $p(U)$ yields the maximum entropy subject to a set of constraints on the PDF. The range of possible constraints is limitless in general; however we will examine the case where the first $n$ central moments have given values.

We will note that an implied constraint always applies to any PDF, that is

$$
\int_{-\infty}^{\infty} p(U) d U=1
$$

We also observe that the mean value of $U$ is

$$
\bar{U}=\int_{-\infty}^{\infty} U p(U) d U
$$

which is not equal to zero in general. The central moments are obtained by subtracting the mean value to give a new set of continuous random variable given by $u=U-\bar{U}$. This yields the following formula for the $i^{\text {th }}$ moment

$$
\mu_{i}=\int_{-\infty}^{\infty} u^{i} p(u) d u
$$

Here we note that the previous discussion indicates that $\mu_{0}=1$ and $\mu_{1}=0$ as basic minimum constraints for any PDF.

We will examine the case which constrains the moments up to the $4^{\text {th }}$ order, with the additional requirement that $u$ has bounded support between $-a$ and $a$. The entropy of the PDF is now given by

$$
s=-\int_{a}^{b} p \ln p d u .
$$

The determination of the PDF that maximizes this integral requires us to turn to the calculus of variation. The PDF that yields the extreme value of the entropy requires us to solve Euler's equation

$$
\frac{\partial \Phi}{\partial y}=0
$$


where $\Phi=-p \ln p+\lambda_{0}^{\prime} C_{0}+\lambda_{1} C_{1}+\lambda_{2} C_{2}+\lambda_{3} C_{3}+\lambda_{3} C_{3} \quad$ where the $\lambda$ are constants. Hence the equation for $p$ is obtained from

$$
\frac{\partial \Phi}{\partial p}=-\ln (p)+1+\lambda_{0}^{\prime}+\lambda_{1} u+\lambda_{2} u^{2}+\lambda_{3} u^{3}+\lambda_{4} u^{4}=0 .
$$

The general form of $p$ is thus given by

$$
\begin{aligned}
p & =\exp \left(1+\lambda_{0}+\lambda_{1} u+\lambda_{1} u^{2}+\lambda_{3} u^{3}+\lambda_{4} u^{4}\right) \\
& =\exp \left(\lambda_{0}+\lambda_{1} u+\lambda_{1} u^{2}+\lambda_{3} u^{3}+\lambda_{4} u^{4}\right) \\
& =\exp (F(u))
\end{aligned}
$$

where $\lambda_{0}=1+\lambda_{0}^{\prime}$.

\section{Solving for the coefficients}

Analytical determination of the coefficients for the maximum entropy approximation to the PDF requires us to obtain closed form integrals of the moments. Many of these integrals are known to be unknown, so analytical solution is not possible. Hence we must resort to a numerical scheme to solve for the coefficients. The method that was used was based on Newton's method. A linear approximation of any of the moments can be written as follows for incremental changes in the coefficients

$$
\mu_{i}-\hat{\mu}_{i}=\frac{\partial \hat{\mu}}{\partial \lambda_{0}} d \lambda_{0}+\frac{\partial \hat{\mu}}{\partial \lambda_{1}} d \lambda_{1}+\frac{\partial \hat{\mu}}{\partial \lambda_{2}} d \lambda_{2}+\frac{\partial \hat{\mu}}{\partial \lambda_{3}} d \lambda_{3}+\frac{\partial \hat{\mu}}{\partial \lambda_{4}} d \lambda_{4} .
$$

The hat indicates that the moments are for an initial, guessed set of coefficients $\hat{\lambda}_{i}$. Examination of the partial derivatives shows that they have a particularly simple form,

$$
\frac{\partial \hat{\mu}_{i}}{\partial \lambda_{j}}=\int_{a}^{b} x^{i}\left(x^{j} \exp (F(x))\right) d x=\hat{\mu}_{i+j} .
$$

Hence we obtain the following system of linear equations given by

$$
\left[\begin{array}{ccccc}
\hat{\mu}_{0} & \hat{\mu}_{1} & \hat{\mu}_{2} & \hat{\mu}_{3} & \hat{\mu}_{4} \\
\hat{\mu}_{1} & \hat{\mu}_{2} & \hat{\mu}_{3} & \hat{\mu}_{4} & \hat{\mu}_{5} \\
\hat{\mu}_{2} & \hat{\mu}_{3} & \hat{\mu}_{4} & \hat{\mu}_{5} & \hat{\mu}_{6} \\
\hat{\mu}_{3} & \hat{\mu}_{4} & \hat{\mu}_{5} & \hat{\mu}_{6} & \hat{\mu}_{7} \\
\hat{\mu}_{4} & \hat{\mu}_{5} & \hat{\mu}_{6} & \hat{\mu}_{7} & \hat{\mu}_{8}
\end{array}\right]\left[\begin{array}{c}
d \lambda_{0} \\
d \lambda_{1} \\
d \lambda_{2} \\
d \lambda_{3} \\
d \lambda_{4}
\end{array}\right]=\left[\begin{array}{c}
1-\hat{\mu}_{0} \\
\mu_{1}-\hat{\mu}_{1} \\
\mu_{2}-\hat{\mu}_{2} \\
\mu_{3}-\hat{\mu}_{3} \\
\mu_{4}-\hat{\mu}_{4}
\end{array}\right] .
$$

The initial values of the $\lambda_{j}{ }^{\prime} s$ are estimated or guessed and the values of the $\hat{\mu}^{\prime} s$ are numerically computed. Then the values of the $d \lambda_{j}$ are computed from this equation, finally they are added to the guessed values and the process is 
repeated if need be. This iterative procedure can be stopped when either the coefficients of the polynomial converge to within some preset precision or the estimated moments are accurate to within some preset value.

In the numerical scheme that is reported, the iteration were repeated if the magnitude of the $d \lambda$ vector divided by the magnitude of the $\lambda$ vector was less than $10^{-4}$. Computing the coefficients to a greater accuracy was not examined. A similar scheme for terminating the iterations can be based on the computed moments.

This presentation of the numerical method to solve for the maximum entropy problem minimizes some of the numerical problems that have been encountered. First the initial values of the coefficients must be chosen with some care for large order systems. The starting guesses that were examined were to make all of the coefficients equal to zero, but this caused the initial matrix to be a Hilbert matrix. Unfortunately, Hilbert matrices have the well documented property of being ill conditioned. Hence, for a large order approximation, order say 10 or more, we might not be able to even start the iteration process. If the order of the system is smaller we can however always use an initial guess that the coefficients are zero. Notice that setting the initial values of the coefficients to zero is equivalent to guessing that the PDF is uniform on the interval $[a, b]$.

The second difficulty that was encountered is that of computing the moments. For a distribution that is very smoothly distributed across the interval, virtually any numerical integration scheme will work. The computed moments will be accurate with relatively little work. However, if the distribution is very spiky, approaching that of a delta function, the integration will require either an inordinate number of intervals or some sort of adaptive method. Simpson's method with 1000 subintervals was selected to compute the moments for the results given here. It is quite clear that some distributions will tax the accuracy of this kind of integration scheme to the point of making the iterations unstable.

The final difficulty that was encountered is that of solving the system of equations. For smooth distributions, it has been observed that the system of equations is relatively well conditioned, and that the system can be solved using Gauss-Jordan elimination. For very spiky distributions, the system of equations is not well conditioned, and the iterative procedure is then unstable. Singular value decomposition must be used to deal with this ill-condition of the matrix. The singular value decomposition algorithm that was used for this work is that given by Press et al. [2]. The values of the terms of the diagonal matrix were set to zero if they were less that $\mathrm{P}$ times the maximum value of the diagonal. By experiment, it was found that $\mathrm{P}=10^{-12}$ was the minimum value of this factor that would allow the iterations to proceed stably for all cases that were examined. If $\mathrm{P}$ was chosen too large, a solution could be obtained but it would be very misleading. Results of a numerical experiment for values of $\mathrm{P}$ that ranged from $10^{-6}$ to $10^{-12}$ clearly showed the effect of $\mathrm{P}$ being too large.

As a concluding remark on the numerics of the maximum entropy approximation it should be clearly stated that the method does work and it appears to work quite well. However, there are some difficulties that must be carefully addressed in any algorithm that is going to be used to obtain the 
coefficients of the polynomial. To repeat, they are the numerical integration of the moments, selection of the initial values of the coefficients and ill-condition of the generated system of equations. However, these problems can be satisfactorily resolved if they are directly addressed.

As a note on the computational efficiency of this scheme we should state that most of the computer time was spent performing the integrations. If the scheme is to be made more efficient, we would have to find computationally more efficient means of performing the integrations. Solving the system of equations, updating the coefficients and testing for convergence generally took less than evaluating even one of the moments. The number of iterations that must be performed is strongly dependent upon the initial guess of the coefficients and the final shape of the PDF. For very narrow distributions close to zero, the scheme required in the order of 50 iterations to converge. It can be hypothesized that better starting values could reduce the iteration count significantly. However, there is no a priori means of determining the initial guess. If one of the moments is tracked as the iterations proceed it will appear that the process converges, diverges and then converges again. This is due to all of the moments being fit so the behavior of one moment may appear oscillatory.

\section{The maximum entropy for four degrees of freedom}

Assume that $p(U, V, W, \Psi)$ is the probability density function describing the turbulent flow as a function of the velocity components $(U, V$, and $W$ ) and the pressure, $\Psi$. Then the statistical entropy for this PDF is given by

$$
s=-\int_{-\infty}^{\infty} \int_{-\infty}^{\infty} \int_{-\infty}^{\infty} \int_{-\infty}^{\infty} p(U, V, W, \Psi) \ln p(U, V, W, \Psi) d U d V d W d \Psi .
$$

For a given set of conditions, $p(U, V, W, \Psi)$ is the function that maximizes $s$. Based on physical considerations we would expect that $p \rightarrow 0$ when any of the variables go to $\pm \infty$.

The basic requirement for a PDF is that

$$
\int_{-\infty}^{\infty} \int_{-\infty}^{\infty} \int_{-\infty}^{\infty} \int_{-\infty}^{\infty} p(U, V, W, \Psi) d U d V d W d \Psi=1
$$

We will employ $(N+1)^{4}$ constraints on the statistical moments about the mean, given by

$$
C_{i, j, k, l}=\int_{-\infty}^{\infty} \int_{-\infty}^{\infty} \int_{-\infty}^{\infty} \int_{-\infty}^{\infty} u^{i} v^{j} w^{k} \psi^{l} p(u, v, w, \psi) d u d v d w d \psi
$$

where the lower case indicates the fluctuation from the mean, and the $i, j, k$, and $l$ are any integers from 0 through $N$ inclusive. 
The known solution to this problem (Weinstock [3]) is obtained by forming the function

$$
f^{*}=-p \ln p-\sum_{i=0}^{N} \sum_{j=0}^{N} \sum_{k=0}^{N} \sum_{l=0}^{N} u^{i} v^{j} w^{k} \psi^{l} \lambda_{i j k l} p
$$

and solving for

$$
\frac{\partial f^{*}}{\partial p}=0 .
$$

This yields

$$
\frac{\partial f^{*}}{\partial p}=-\ln p-1-\sum_{i=0}^{N} \sum_{j=0}^{N} \sum_{k=0}^{N} \sum_{l=0}^{N} u^{i} v^{j} w^{k} \psi^{l} \lambda_{i j k l}=0
$$

Thus $p$ is given by

$$
\ln p=1-\sum_{i=0}^{N} \sum_{j=0}^{N} \sum_{k=0}^{N} \sum_{l=0}^{N} u^{i} v^{j} w^{k} \psi^{l} \lambda_{i j k l} .
$$

Hence the maximum entropy PDF for the stated constraints is given by

$$
p=\exp \left(1-\sum_{i=0}^{N} \sum_{j=1}^{N} \sum_{k=0}^{N} \sum_{l=0}^{N} u^{i} v^{j} w^{k} \psi^{l} \lambda_{i j k l}\right) .
$$

The constants, $\lambda_{i j k l}$ are set so the final PDF has the same moments as given by the constraints.

\section{Experimental data}

Experimental data for one-dimensional moments of turbulent flows of order 5 or higher are very difficult to find and tend to come from work published in the 1970's. The sources of experimental data used for this work come from the following sources. Antonia and Sreenivasan [4] presented moments up to $8^{\text {th }}$ order of the axial and cross-stream velocities and temperature for a turbulent heated jet. Py and Duhamel [5] presented moments up to the $5^{\text {th }}$ order moment for the partial derivative of the velocity at the wall as a function of Reynolds number for a two-dimensional channel. The moments up to the $6^{\text {th }}$ order of the fluctuating wall shear stress for an eight degree conical were reported by Derksen [6].

\subsection{Comparison to the maximum entropy approximation}

The maximum entropy approximation was applied to the experimental data using the measured moments up to and including the fourth as constraints. In all cases, the final predicted movements up to and including the fourth matched to better than four decimal places, confirming that the approximation was correct. 
Table 1: $\quad$ The maximum entropy and measured fifth order moments.

\begin{tabular}{|c|c|c|c|c|c|}
\hline Source & Meas. & Comp. & Source & Meas. & Comp. \\
\hline $\begin{array}{l}\text { Antonia, } \\
u, \eta=0.00\end{array}$ & -1.52 & -1.34 & $\begin{array}{l}\mathrm{Py}, \tau_{w} \\
\mathrm{Re}=1935\end{array}$ & 120 & 117 \\
\hline $\begin{array}{l}\text { Antonia, } \\
u, \eta=0.67\end{array}$ & 0.17 & 0.22 & $\begin{array}{l}\mathrm{Py}, \tau_{w}, \\
\mathrm{Re}=2300\end{array}$ & 27.9 & 27.7 \\
\hline $\begin{array}{l}\text { Antonia, } \\
u, \eta=0.89\end{array}$ & 2.28 & 2.36 & $\begin{array}{l}\mathrm{Py}, \tau_{w}, \\
\operatorname{Re}=2615\end{array}$ & 5.76 & 4.42 \\
\hline $\begin{array}{l}\text { Antonia, } \\
u, \eta=1.19\end{array}$ & 8.54 & 9.37 & $\begin{array}{l}\mathrm{Py}, \tau_{w}, \\
\mathrm{Re}=3500\end{array}$ & 4.98 & 4.50 \\
\hline $\begin{array}{l}\text { Antonia, } \\
u, \eta=1.48\end{array}$ & 39.3 & 55.2 & $\begin{array}{l}\mathrm{Py}, \tau_{w} \\
\mathrm{Re}=5000\end{array}$ & 4.37 & 4.20 \\
\hline $\begin{array}{l}\text { Antonia, } \\
u, \eta=1.63\end{array}$ & 24.5 & 39.5 & $\begin{array}{l}\mathrm{Py}, \tau_{w}, \\
\mathrm{Re}=7200\end{array}$ & 4.44 & 4.04 \\
\hline $\begin{array}{l}\text { Antonia, } \\
v, \eta=0.00\end{array}$ & -0.53 & -0.96 & $\begin{array}{l}\mathrm{Py}, \tau_{w}, \\
\mathrm{Re}=9950\end{array}$ & 4.25 & 3.90 \\
\hline $\begin{array}{l}\text { Antonia, } \\
v, \eta=0.67\end{array}$ & 1.24 & 1.53 & $\begin{array}{l}\mathrm{Py}, \tau_{w}, \\
\mathrm{Re}=15000\end{array}$ & 4.17 & 3.84 \\
\hline $\begin{array}{l}\text { Antonia, } \\
v, \eta=0.89\end{array}$ & 3.45 & 4.22 & $\begin{array}{l}\text { Derksen, } \tau_{w}, \\
\mathrm{x}=4.62\end{array}$ & 9.80 & 9.10 \\
\hline $\begin{array}{l}\text { Antonia, } \\
v, \eta=1.19\end{array}$ & 9.52 & 13.0 & $\begin{array}{l}\text { Derksen, } \tau_{w}, \\
\mathrm{x}=15.59\end{array}$ & 5.22 & 4.98 \\
\hline $\begin{array}{l}\text { Antonia, } \\
v, \eta=1.48\end{array}$ & 37.7 & 51.2 & $\begin{array}{l}\text { Derksen, } \tau_{w}, \\
\mathrm{x}=19.58\end{array}$ & 3.30 & 2.97 \\
\hline $\begin{array}{l}\text { Antonia, } \\
v, \eta=1.63\end{array}$ & 74.6 & 112 & $\begin{array}{l}\text { Derksen, } \tau_{w}, \\
\mathrm{x}=23.57\end{array}$ & 3.94 & 3.53 \\
\hline $\begin{array}{l}\text { Antonia, } \\
\theta, \eta=0.00\end{array}$ & -3.35 & -3.84 & $\begin{array}{l}\text { Derksen, } \tau_{w}, \\
\mathrm{x}=31.55\end{array}$ & 4.69 & 4.23 \\
\hline $\begin{array}{l}\text { Antonia, } \\
\theta, \eta=0.67\end{array}$ & -1.09 & -1.51 & $\begin{array}{l}\text { Derksen, } \tau_{w}, \\
\mathrm{x}=39.53\end{array}$ & 4.40 & 3.86 \\
\hline $\begin{array}{l}\text { Antonia, } \\
\theta, \eta=0.89\end{array}$ & 0.79 & 0.31 & $\begin{array}{l}\text { Derksen, } \tau_{w}, \\
\mathrm{x}=50.71\end{array}$ & 9.43 & 8.96 \\
\hline $\begin{array}{l}\text { Antonia, } \\
\theta, \eta=1.19\end{array}$ & 3.80 & 3.11 & $\begin{array}{l}\text { Derksen, } \tau_{w}, \\
\mathrm{x}=55.69\end{array}$ & 9.55 & 8.69 \\
\hline $\begin{array}{l}\text { Antonia, } \\
\theta, \eta=1.48\end{array}$ & 20.3 & 18.1 & $\begin{array}{l}\text { Derksen, } \tau_{w}, \\
\mathrm{x}=69.46\end{array}$ & 7.13 & 6.75 \\
\hline $\begin{array}{l}\text { Antonia, } \\
\theta, \eta=1.63\end{array}$ & 70.9 & 64.9 & $\begin{array}{l}\text { Derksen, } \tau_{w}, \\
\text { pipe }\end{array}$ & 1.97 & 1.87 \\
\hline $\mathrm{Py}, \tau_{w}, \mathrm{e}=1700$ & 12.3 & 0.21 & & & \\
\hline
\end{tabular}


The resulting fifth and sixth order moments from the maximum entropy approximation are compared to the measured moments in Table 1 for the fifth order moments and Table 2 for the sixth order moments. General agreement between the measured and computed fifth order moments is observed with two exceptions. These exceptions are: (1) The agreement is poor at the outer edge of the heated jet, $\eta=1.48$ and 1.63 , This may be due to the flow being highly intermittent in this zone. (2) The Py data at the lowest Reynolds numbers, 1700 and 1935, also does not agree well, however the agreement is much better as the Reynolds number increase. So this may be due to the flow not being fully turbulent.

Table 2: $\quad$ The maximum entropy and measured sixth order moments.

\begin{tabular}{|c|c|c|c|c|c|c|}
\hline Source & Meas. & Comp. & Source & & Meas. & Comp. \\
\hline $\begin{array}{l}\text { Antonia, } \\
u, \eta=0.00\end{array}$ & 15.7 & 15.1 & $\begin{array}{l}\text { Antonia, } \\
\theta, \eta=0.89\end{array}$ & & 7.28 & 7.30 \\
\hline $\begin{array}{l}\text { Antonia, } \\
u, \eta=0.67\end{array}$ & 12.6 & 11.9 & $\begin{array}{l}\text { Antonia, } \\
\theta, \eta=1.19\end{array}$ & & 10.0 & 8.07 \\
\hline $\begin{array}{l}\text { Antonia, } \\
u, \eta=0.89\end{array}$ & 14.2 & 12.6 & $\begin{array}{l}\text { Antonia, } \\
\theta, \eta=1.48\end{array}$ & & 72.0 & 55.0 \\
\hline $\begin{array}{l}\text { Antonia, } \\
u, \eta=1.19\end{array}$ & 32.0 & 31.6 & $\begin{array}{l}\text { Antonia, } \\
\theta, \eta=1.63\end{array}$ & & 358 & 282 \\
\hline $\begin{array}{l}\text { Antonia, } \\
u, \eta=1.48\end{array}$ & 234 & 369 & $\begin{array}{l}\text { Derksen, } \\
x=4.62\end{array}$ & $\tau_{w}$ & 41.1 & 36.0 \\
\hline $\begin{array}{l}\text { Antonia, } \\
u, \eta=1.63\end{array}$ & 508 & 878 & $\begin{array}{l}\text { Derksen, } \\
x=15.59\end{array}$ & $\tau_{w}$ & 22.7 & 21.8 \\
\hline $\begin{array}{l}\text { Antonia, } \\
v, \eta=0.00\end{array}$ & 17.6 & 21.6 & $\begin{array}{l}\text { Derksen, } \\
x=19.58\end{array}$ & $\tau_{w}$ & 18.0 & 17.5 \\
\hline $\begin{array}{l}\text { Antonia, } \\
v, \eta=0.67\end{array}$ & 14.3 & 13.7 & $\begin{array}{l}\text { Derksen, } \\
x=23.57\end{array}$ & $\tau_{w}$ & 18.6 & 17.2 \\
\hline $\begin{array}{l}\text { Antonia, } \\
v, \eta=0.89\end{array}$ & 19.7 & 20.3 & $\begin{array}{l}\text { Derksen, } \\
\mathrm{x}=31.55\end{array}$ & $\tau_{w}$ & 20.1 & 18.3 \\
\hline $\begin{array}{l}\text { Antonia, } \\
v, \eta=1.19\end{array}$ & 44.4 & 59.0 & $\begin{array}{l}\text { Derksen, } \\
\mathrm{x}=39.53\end{array}$ & $\tau_{w}$ & 18.4 & 16.5 \\
\hline $\begin{array}{l}\text { Antonia, } \\
v, \eta=1.48\end{array}$ & 223 & 319 & $\begin{array}{l}\text { Derksen, } \\
\mathrm{x}=50.71\end{array}$ & $\tau_{w}$ & 39.7 & 36.4 \\
\hline $\begin{array}{l}\text { Antonia, } \\
v, \eta=1.63\end{array}$ & 594 & 1000 & $\begin{array}{l}\text { Derksen, } \\
\mathrm{x}=55.69\end{array}$ & $\tau_{w}$ & 39.0 & 33.0 \\
\hline $\begin{array}{l}\text { Antonia, } \\
\theta, \eta=0.00\end{array}$ & 18.7 & 21.0 & $\begin{array}{l}\text { Derksen, } \\
x=69.46\end{array}$ & $\tau_{w}$ & 27.6 & 25.7 \\
\hline $\begin{array}{l}\text { Antonia, } \\
\theta, \eta=0.67\end{array}$ & 9.46 & 10.1 & $\begin{array}{l}\text { Derksen, } \\
\text { pipe }\end{array}$ & & 14.3 & 14.0 \\
\hline
\end{tabular}


The results for the sixth moment mirror those for the fifth moment, however it should be noted that no data for the sixth moment was available from the Py data.

These results indicate that the maximum entropy approximation predictions of the fifth and sixth order moments are in general agreement to those obtained from measurement where the flow is fully turbulent. This is an interesting observation but further experimental studies would need to be done to confirm that turbulence generated PDFs are maximum entropy PDFs that constrain moments to the fourth order.

One factor that needs careful consideration in further studies is that of the uncertainty in measuring the higher order moments. This mandates the use of very large samples, in the order of 100,000 to $1,000,000$ samples, to ensure that the rare events can be observed.

\section{Conclusions}

The results presented here present a compelling case for assuming that the PDF from a turbulent process results in a maximum entropy approximation that constrains moments up to the fourth order. However, it is recognized that more study needs to be done to confirm the validity of this statement.

\section{References}

[1] P.W. Atkins, The $2^{\text {nd }}$ Law, Energy, Chaos, and Form, Scientific American Library, New York, 1994.

[2] W.H. Press, B.P. Flannery, S.A. Teukolsky and W.T. Vetterling, Numerical Recipes: The Art of Scientific Computing, Cambridge University Press, Cambridge, 1986, pg 52-64.

[3] R. Weinstock, Calculus of Variations with Applications to Physics and Engineering, Dover, New York, 1974,pg. 134.

[4] R.A. Antonia and K.R. Sreenivasan, Statistical Properties of Velocity and Temperature Fluctuations in a Turbulent heated Jet, University of Newcastle Report, T.N.F.M. (1976).

[5] B. Py, and P. Duhamel, Mesure des Distributions Statistiques du Gradient Parietal de Vitesse Longitudinale en Canal Bidimensional, C.R. Acad. Sc. Paris 275 (1972) 373-376.

[6] R.W. Derksen, An Experimental Investigation of the Fluctuating Wall Shear Stresses in an Eight Degree Conical Diffuser, Ph.D. thesis, University of Manitoba, Winnipeg, MB, Canada, 1985. 